\title{
Long-Standing Persistent Atrial Fibrillation Ablation: How Do You Perform? Left and Right Atrial Linear Ablation in Addition to Pulmonary Vein Isolation
}

Michele Brunelli1,*, Mark Adrian Sammut ${ }^{2}$

\section{ORCID IDs}

Brunelli M (D) https://orcid.org/0000-0002-5960-8374

Sammut MA (D) https://orcid.org/0000-0001-7274-7689

\begin{abstract}
Catheter ablation of long-standing persistent atrial fibrillation is not yet clearly defined with respect to endpoints, and different ablative strategies are offered to patients. Presented here is an approach aiming at biatrial debulking in the form of extensive linear ablation, specifically targeting areas of low-voltage complex fractionated electrograms, in addition to pulmonary vein isolation. Its main advantage is that it is not dependent on operator/system variability, since the strategy of isolating the pulmonary veins, superior vena cava and left atrial posterior wall together with achievement of bidirectional block during linear ablation provides objective endpoints that can consistently be reproduced.
\end{abstract}

KEYWORDS: Radiofrequency catheter ablation; Atrial fibrillation.

1. Klinikum Magdeburg - Department of Cardiology and Diabetology Section of Clinical and Invasive Cardiac Electrophysiology Magdeburg - Germany

2.Mater Dei Hospital - Department of Cardiology - Msida - Malta

*Corresponding author: mbrune78@yahoo.com

Received: 18 Jun 2020 | Accepted: 20 Jun 2020 


\section{INTRODUCTION}

Morbidity and mortality associated with atrial fibrillation (AF) has been described for the first time 4000 years ago in The Yellow Emperor's Classic of Internal Medicine. Although during the last three decades a large body of research has studied and confirmed beyond doubt the benefit of treatment for $\mathrm{AF}$, including appropriate (oral) anticoagulation ${ }^{1}$ and rhythm control with catheter ablation in specific patients ${ }^{2}$, a clear consensus regarding extension, goals, and energy source of ablation in (long-standing) persistent $\mathrm{AF}$ is still missing.3. With this case report an approach using left and right atrial linear ablation in addition to pulmonary vein isolation for catheter ablation of long-standing persistent $\mathrm{AF}$ is presented.

\section{CASE REPORT}

A 64-year old gentleman with recurrent symptomatic (EHRA IV) episodes of persistent AF (initially diagnosed in 2002, CHA2DS2-Vasc 5, HASBLED 2), previous biological aortic valve replacement (Sorin Solo 25) and concomitant coronary-artery bypass graft surgery (left internal mammary to anterior descending coronary artery) for severe aortic stenosis and coronary artery disease (August 2012), a reduced ejection fraction on medical treatment for heart failure (HF), arterial hypertension, dyslipidemia and diabetes, underwent several (\#5) electrical cardioversions that were invariably followed by relapses (associated with sudden worsening of HF) in spite of chronic amiodarone therapy. The patient had a cardiac resynchronization therapy pacemaker (Quadra Allure, Abbott) implanted due to concomitant right bundle and left anterior (hemi)-block in April 2019 and was then scheduled, 12 months after the last cardioversion, to undergo catheter ablation.

During hospital admission (May 2019), the day before catheter ablation, the patient was in AF (EHRA III), the left atrium was dilated $(49 \times 64 \mathrm{~mm})$, and transesophageal echocardiography excluded cardiac thrombi and showed moderate functional mitral and tricuspid insufficiency. He couldn't undergo cardiac imaging (i.e. cardiac computed tomography or magnetic resonance imaging) due to chronic renal failure (glomerular filtration rate $33 \mathrm{~mL} / \mathrm{min}$ ). On the day of the procedure (international normalized ratio 2.77, uninterrupted Phenprocoumon), after administration of intravenous midazolam, fentanyl and propofol for deep sedation, bilateral femoral access was taken, 2 decapolar steerable Inquiry catheters were, with limited use of X-rays (due to the presence of pace-maker leads), positioned at the His (large curve) and in the coronary sinus (CS, medium curve, both Abbott). Under fluoroscopic guidance (Siemens, Germany) 2 long sheaths (steerable Agilis and SL1, St. Jude Medical, St. Paul, MN, USA) were placed in the left atrium (LA), and systemic anticoagulation with intravenous heparin was started with an initial bolus of $100 \mathrm{U} / \mathrm{kg}$ (and additional doses to achieve and maintain an ACT value of $\approx 350 \mathrm{~s}$ ). At this point fluoroscopy was turned off (total time $2.3 \mathrm{~min}$, total dose $\sim 100 \mu \mathrm{G} / \mathrm{m} 2$ ), all the staff removed the lead apron, and a LA geometry was collected with the Advisor HD-Grid sensor enabled catheter and the Ensite Precision 3D mapping system (both Abbott).

Mapping, in AF, showed diffuse area of low voltage (0.5-0.05 mV) with fractionated electrograms (EGMs, 3 deflections) concentrated on the posterior (PW) and the superoseptal LA wall (Fig. 1). Wide ipsilateral catheter ablation (TactiCath-65, Abbott, $35 \mathrm{~W}, 41^{\circ}, \sim 17 \mathrm{~mL} / \mathrm{h}$ ) of the pulmonary veins (PVs) was performed targeting LSI $4-5$ on the posterior wall (PW), increasing up to 5 in other areas, reaching $\sim 6$ at sequence change or isolation (Fig. 2).

The catheter location was slightly shifted when the esophageal temperature increased to $>39^{\circ}$ (CIRCA S-CATH, CIRCA Scientific) and ablation was interrupted for temperatures $>41^{\circ}$. Areas of faster atriopulmonary vein conduction were first targeted with radiofrequency $(\mathrm{RF})$ lesions, so that isolation was achieved as quickly as possible and waiting time maximized. Next, (anatomical) linear ablation at the LA roof was performed, the HD-Grid catheter then placed at the PW, and linear ablation between the 2 inferior PVs, at first targeting changes in the activation sequence, was added to achieve electrical isolation of the PW (Fig. 3). Since the map had revealed an area of low voltage on the superior septum, a mitral isthmus line connecting the annulus with the middle of the roof line along the septal side of the left atrial appendage and superior wall was plotted with a continuous series of automatic 3D tags of RF lesions (Fig. 4) 


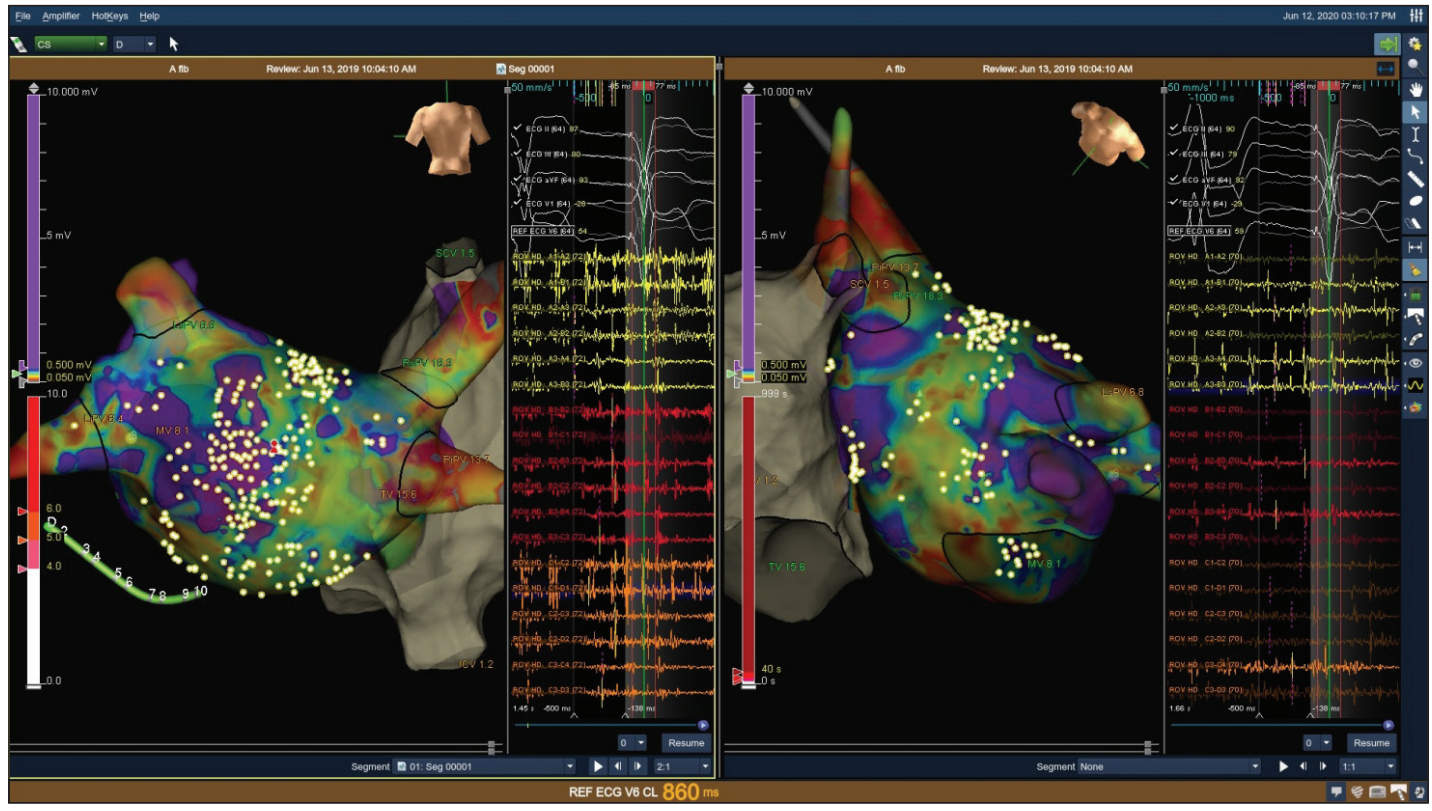

Figure 1. Left atrial map during atrial fibrillation. The image shows diffuse low voltage (0.5-0.05 mV) and fractionated electrograms (white dots, three deflections) especially at the posterior and superoseptal wall of the left atrium. A steerable decapolar Inquiry catheter (green) in the coronary sinus is seen (left). Posteroanterior (left) and cranial left anterior oblique $50^{\circ}$ (right) views are shown.

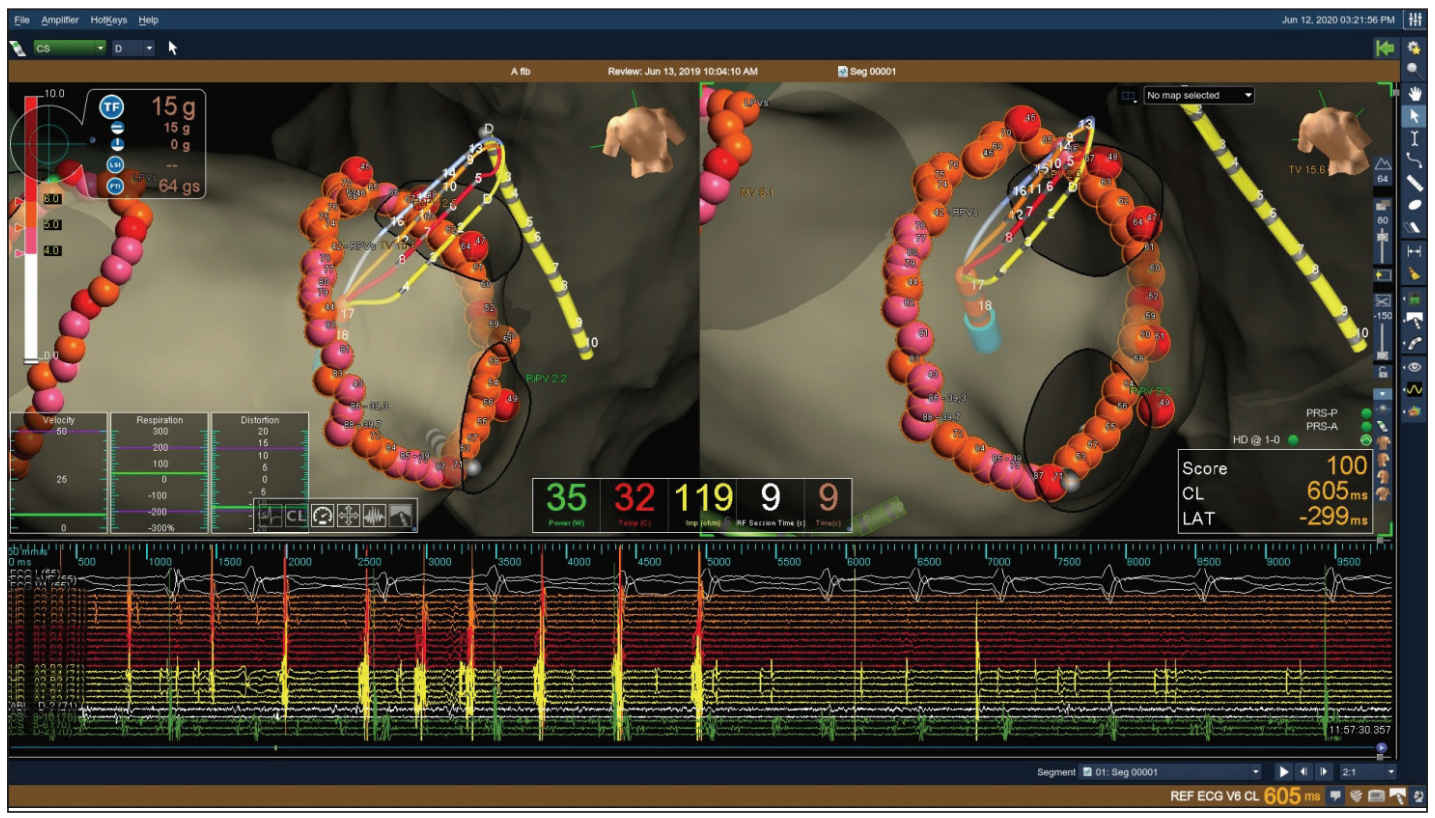

Figure 2. Pulmonary vein isolation. Achievement of right sided pulmonary vein isolation (disappearance of potentials on the HD-Grid placed in the right superior vein) during ablation (TactiCath-65, both Abbot) at the inferoseptal border of the line. Areas of fast atriopulmonary vein conduction were first targeted, so that isolation was achieved as quickly as possible and waiting time maximized, thereafter the ablation line was completed. Two steerable decapolar Inquiry catheters in the coronary sinus (green) and along the right atrial septum (yellow) are seen. Posteroanterior (left) and right posterolateral (right) views are shown. 


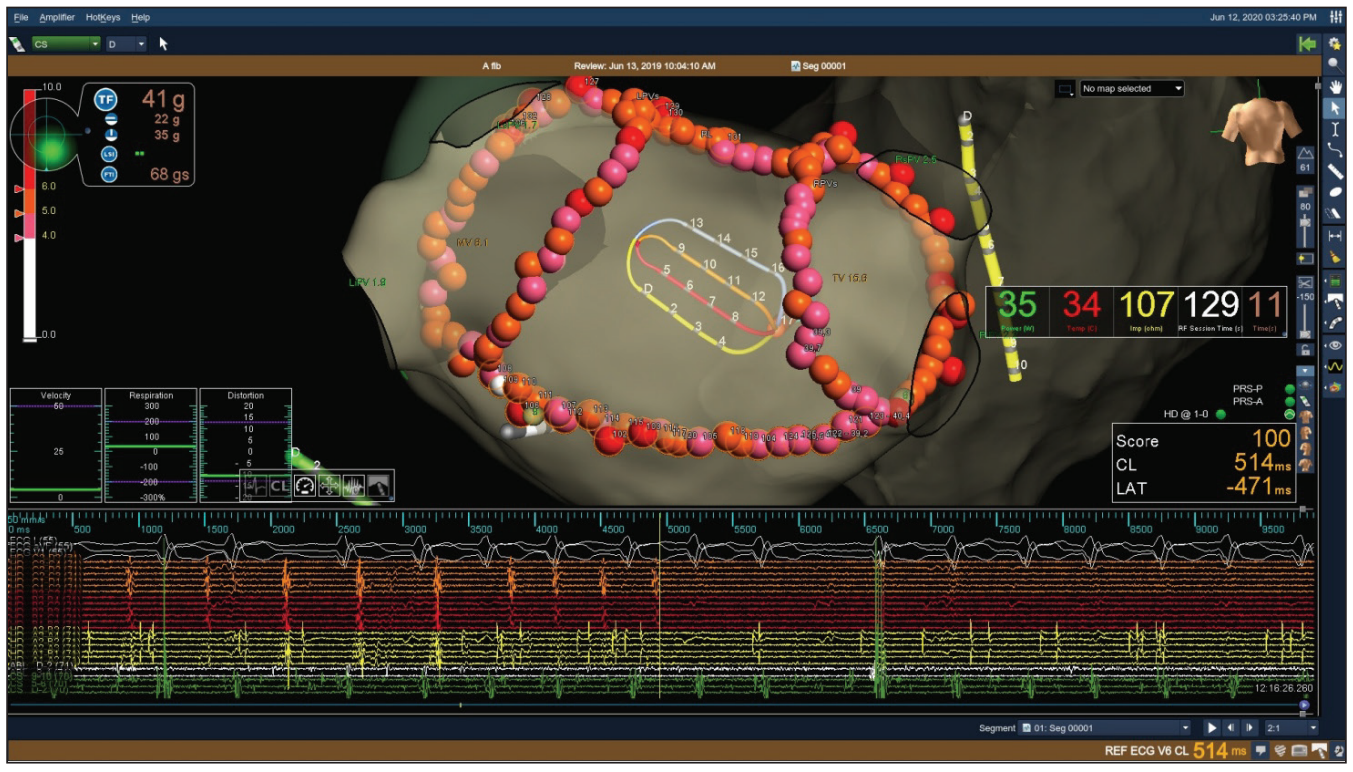

Figure 3. Posterior wall isolation. After linear ablation at the roof, posterior wall isolation (showed by disappearance of electrograms on the HD-Grid catheter) was achieved with radiofrequency lesions (TactiCath-65, $35 \mathrm{~W}, 41^{\circ}$ ) connecting the two inferior pulmonary veins. Lesions were strategically delivered to achieve isolation as quickly as possible, followed by completion of the isolation line, therefore increasing waiting time. Two steerable decapolar Inquiry catheters in the coronary sinus (green) and along the right atrial septum (yellow) are seen. A posterior view is shown.

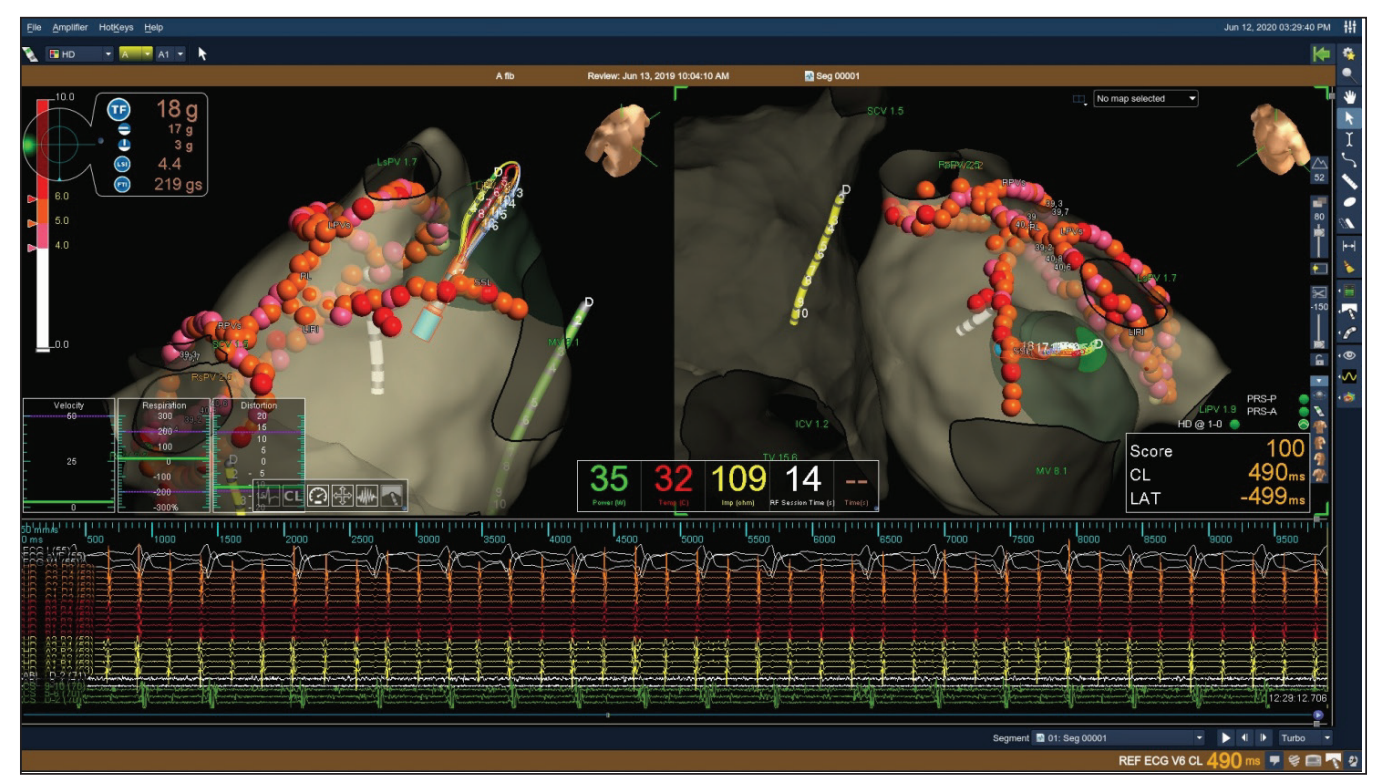

Figure 4. Superoseptal mitral isthmus line. During atrial fibrillation linear ablation (TactiCath-65) between the mitral annulus and the right superior pulmonary vein is plotted. A continuous series of automatic 3D-tags targeting an LSI > 5 increases, although does not imply, the likelihood of demonstrating bidirectional block once sinus rhythm is achieved. HD-Grid catheter in the left atrial appendage. Two steerable decapolar Inquiry catheters in the coronary sinus (green) and along the right atrial septum (yellow) are seen. Cranial right anterior oblique view $30^{\circ}$ (left) and cranial left anterior oblique view $50^{\circ}$ (right) views are shown.

Ablation of fractionated low-voltage EGMs on the posterior-half of the septum, aiming at disappearance of the high frequency components and/or additional isolation of low-voltage areas was then added (Fig. 5). Finally, the right atrium was mapped, and since low-voltage fractionated EGMs were detected on the PW, a continuous series of automatic 3D-tags of RF lesions connecting the superior with the inferior vena cava along the area of highest 
fractionation and sparing areas of diaphragmatic capture was plotted (Fig. 6). In addition, the superior vena cava was isolated (Fig. 6). Since sinus rhythm had still not been achieved, synchronized electrical cardioversion (270J) was performed and conduction block across the cavotricuspid isthmus line was achieved during inferolateral right atrial pacing (Fig. 7).

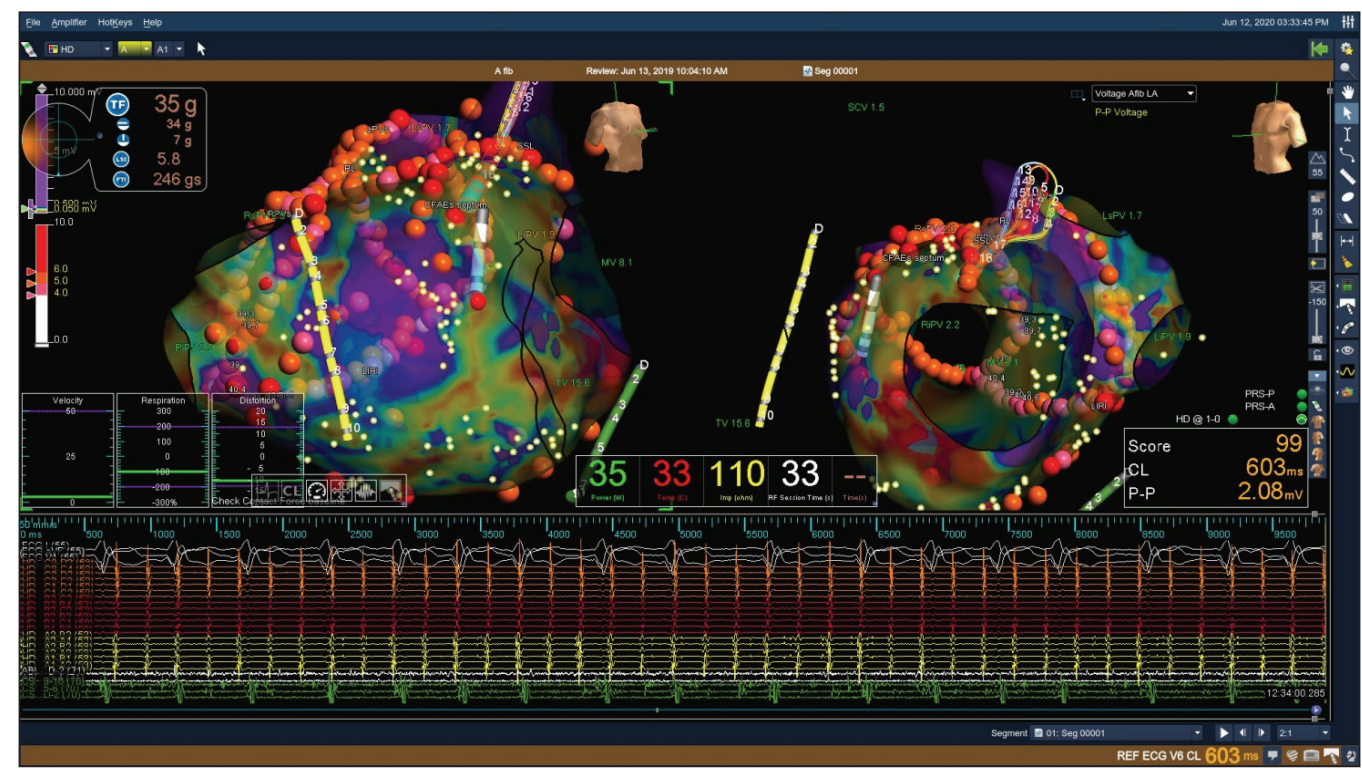

Figure 5. Ablation of complex fractionated atrial electrograms (EGMs). After having (anatomically) completed the superoseptal line, further ablation is directed at fractionated low-voltage EGMs on the posterior-half of the septum, aiming at disappearance of the high frequency components and/or additional isolation of low-voltage areas. HD-Grid catheter into the left atrial appendage. Two steerable decapolar Inquiry catheters in the coronary sinus (green) and along the lateral right atrial wall (yellow) are seen. Right anterior oblique view $30^{\circ}$ (left) and left anterior oblique view $50^{\circ}$ (right) views are shown.

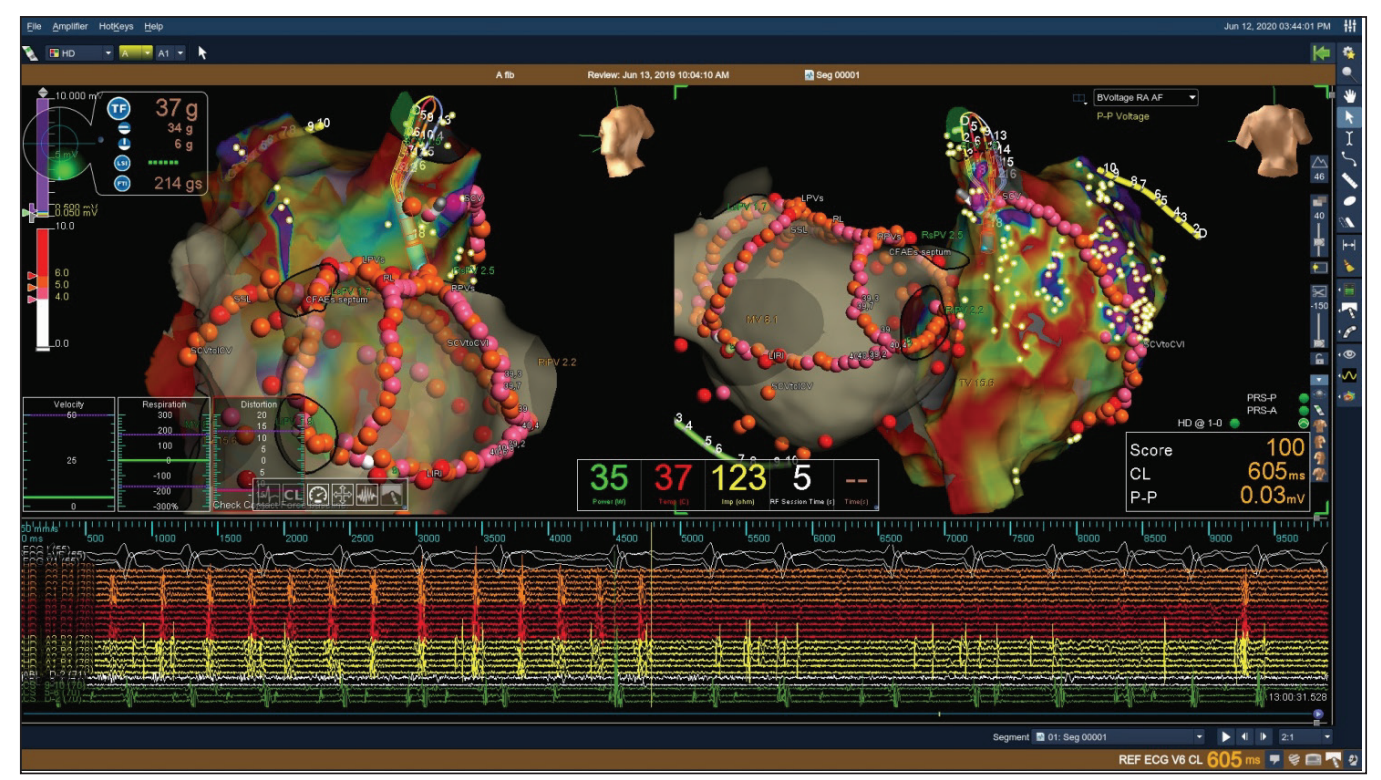

Figure 6. Right atrial mapping and ablation. Voltage mapping of the right atrium showing a broad area of fractionated low-voltage electrograms on the posterior wall. Linear ablation between the superior and inferior vena cava, along the area with the highest fractionation and sparing areas of diaphragmatic capture is performed with a continuous series (0.5-0.05 mV) of automatic 3D-tags targeting an LSI 4-5 increases the likelihood, although does not imply, demonstration of bidirectional block once sinus rhythm is achieved. Thereafter isolation of the superior vena cava (as shown by disappearance of potentials in the HD-Grid placed inside the vein) is carried out. Two steerable decapolar Inquiry catheters in the coronary sinus (green) and along the lateral right atrial wall (yellow) are seen. Left lateral view (left) and right posteroanterior (right) views are shown. 


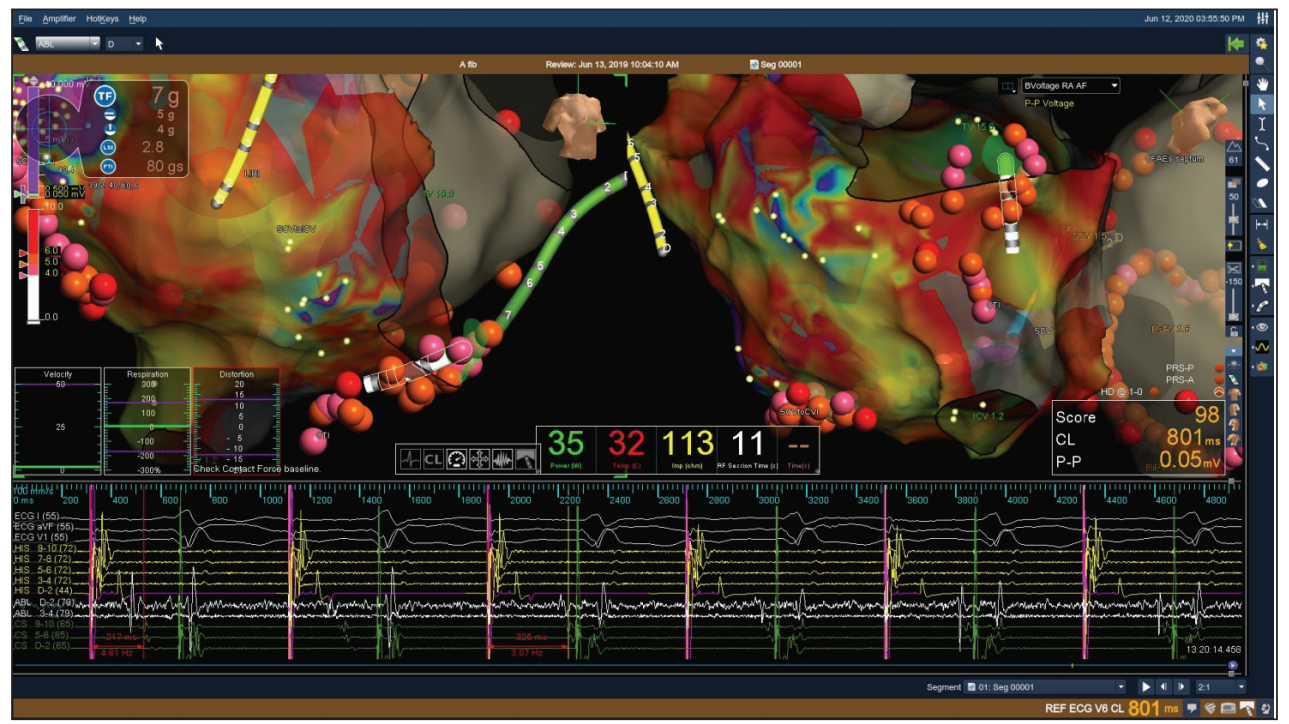

Figure 7. Achievement of block during cavotricuspid isthmus line ablation. Achievement of lateral to septal conduction block (shown by sudden prolongation of conduction time seen both at the electrograms of the ablation catheter and coronary sinus) at the cavotricuspid isthmus while pacing the inferolateral right atrial wall. Once block is achieved the line is then completed with a continuous series of automatic 3D radiofrequency tags. Two steerable decapolar Inquiry catheters in the coronary sinus (green) and along the lateral right atrial wall (yellow) are seen. Right anterior oblique view $30^{\circ}$ (left) and caudal left anterior oblique view $50^{\circ}$ (right) views are shown.

In sinus rhythm, entry- and exit-block (also under adenosine bolus, $30 \mathrm{mg}$ ) of the PVs, the LA PW and the superior vena cava (SVC) were confirmed, and bidirectional block for all three lines (Figs. 8,9 and 10) demonstrated, and further verified with additional differential pacing maneuvers. The procedure lasted $185 \mathrm{~min}$, with $68 \mathrm{~min}$ of RF energy. In order to avoid lead dislodgment, fluoroscopy was again used during right atrial mapping and ablation, and this resulted in a relatively long total fluoroscopy time of $7.8 \mathrm{~min}$ (238 $\mu \mathrm{G} / \mathrm{m} 2,0.5$ millisievert). After the procedure, amiodarone was stopped, and for the first 6 months no recurrences were detected.

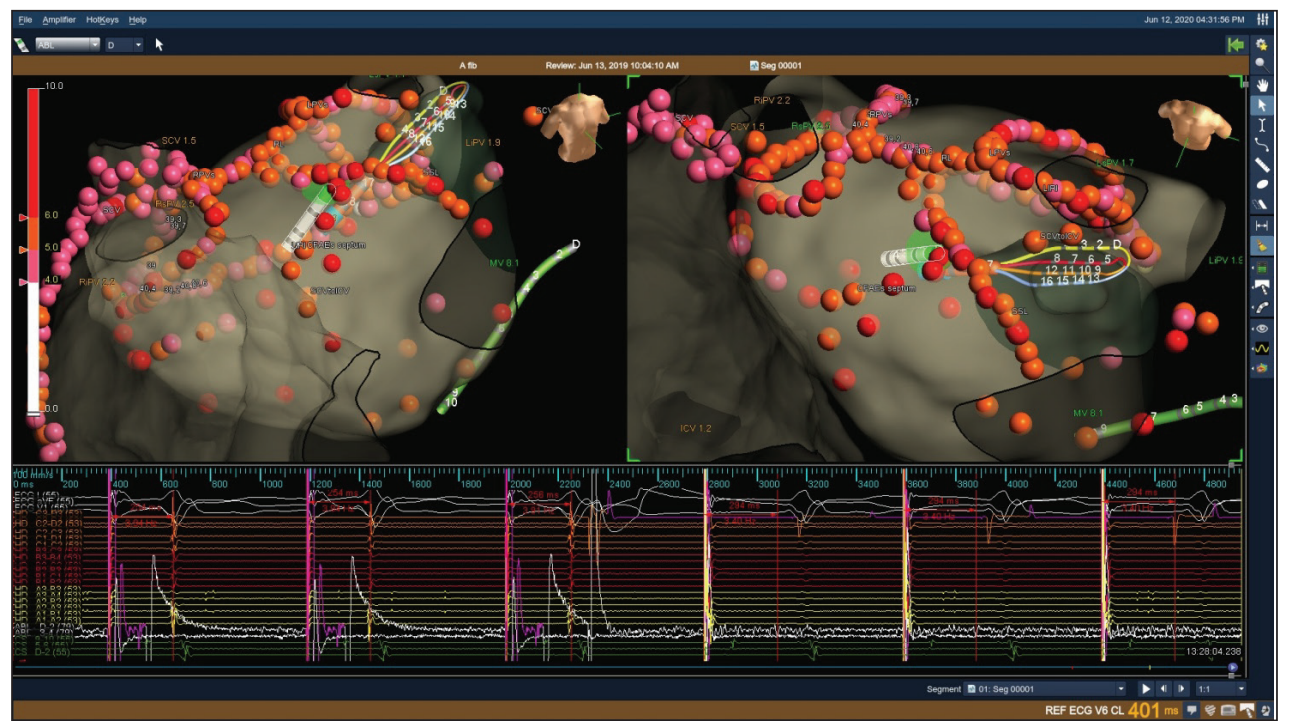

Figure 8. Bidirectional block after mitral isthmus linear ablation at the superior septum (between the annulus and the middle of the roof line along the septal side of the left atrial appendage). Pacing inferoseptal of the line (first 3 beats) reveals prolonged conduction time $(\approx 255 \mathrm{~ms})$ with a lateral to septal activation of the HD-Grid catheter placed in the left atrial appendage. Pacing from the left atrial appendage (last 3 beats) shows a prolonged conduction $(\approx 295 \mathrm{~ms}$ ) time to the ablation catheter placed inferoseptal of the line (low-voltage electrograms partially obscured by the calipers). Activation sequence along the septum (cranial to caudal, anterior to posterior) and differential pacing further proves integrity of ablation line. A steerable decapolar Inquiry catheter (green) in the coronary sinus is seen. Posteroanterior (left) and right posterolateral (right) views are shown. 


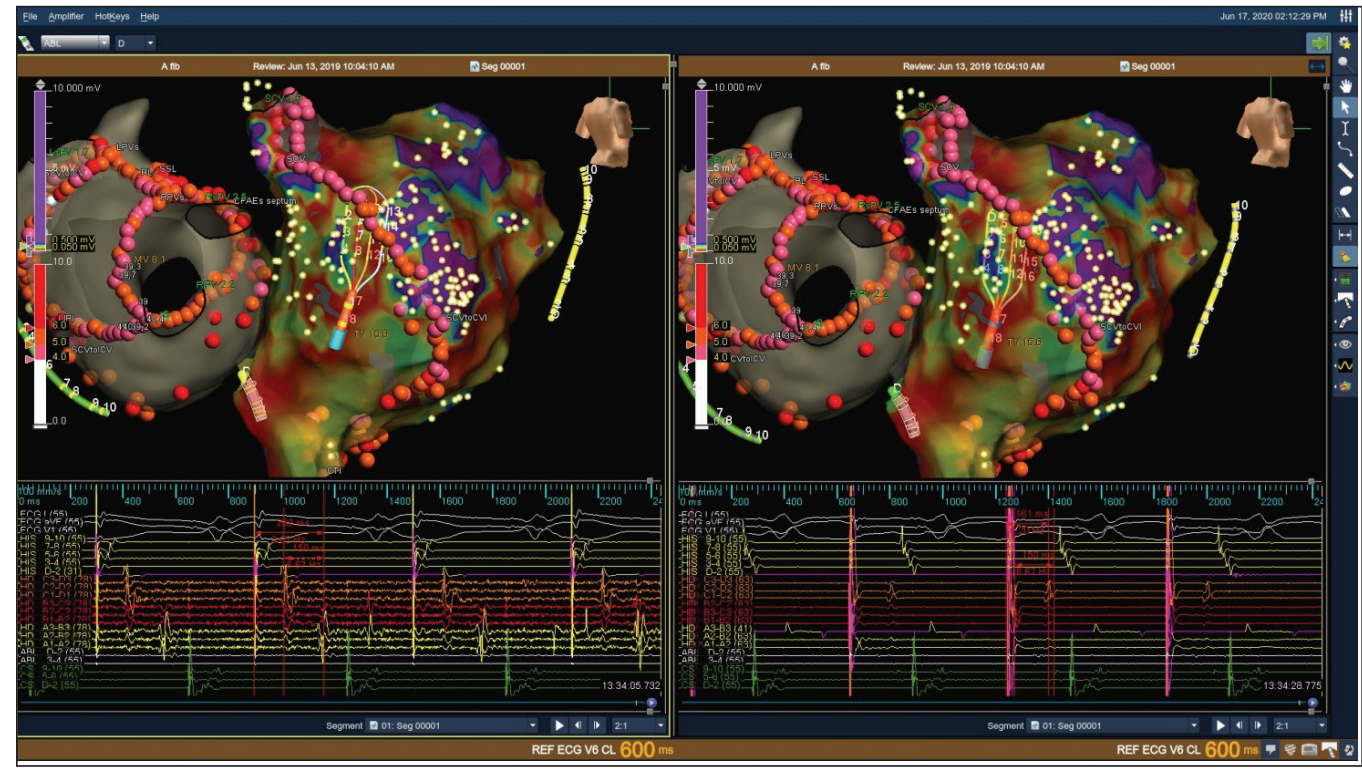

Figure 9. Bidirectional block along the ablation line on the posterior right atrial wall. This is demonstrated by the presence of widely spaced double potentials and long conduction time on the HDGrid catheter while pacing from the septum with the TactiCath-65 (150 and $200 \mathrm{~ms}$, respectively) and the lateral wall with the Inquiry (150 and $161 \mathrm{~ms}$, respectively). Right posterolateral views are shown.

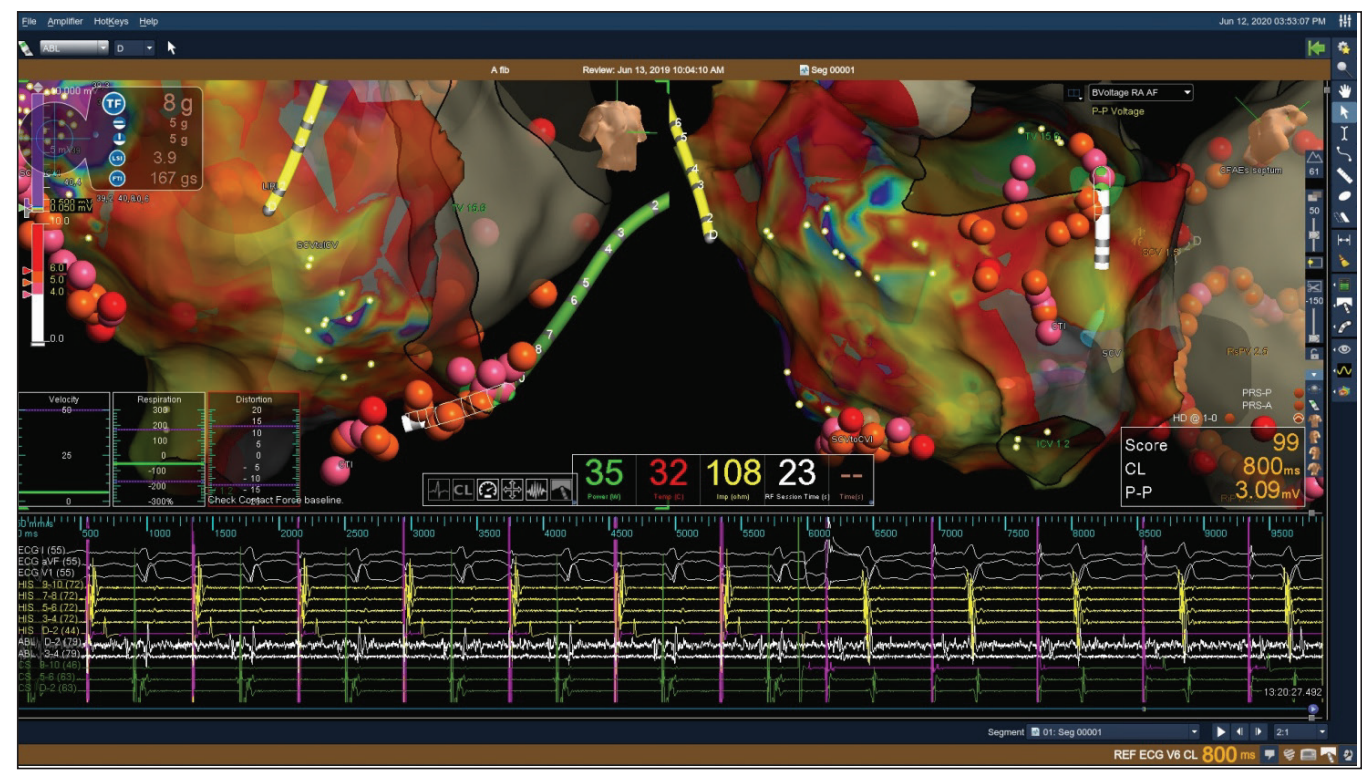

Figure 10. Bidirectional block along the cavotricuspid isthmus line. This is demonstrated by the presence of widely spaced double potentials and long conduction time on the ablation catheter (Tacticath) while pacing from the inferolateral right atrial wall (last 5 beats, 270 and $320 \mathrm{~ms}$, respectively) and the coronary sinus (first 7 beats, 180 and 300 ms, respectively). In addition, a superior to inferior activation on the Inquiry catheter placed on the lateral wall is observed while pacing from the coronary sinus. Calipers are not shown. Right anterior oblique view $30^{\circ}$ (left) and caudal left anterior oblique view $50^{\circ}$ (right) views are shown.

In January 2020, the patient was again admitted with a new episode of symptomatic AF (EHRA III), and electrical cardioversion (2 days after the onset of symptoms) performed. At the beginning of March 2020, the patient again underwent catheter ablation, where persistent isolation of all pulmonary veins and of the superior vena cava was confirmed, and all lines still showed bidirectional block.

Sinus rhythm voltage maps of the left atrium showed an area of low-amplitude $(0.5-0.05 \mathrm{mV})$ fractionated electrograms at the inferolateral mitral isthmus, therefore high power short duration $\left(60 \mathrm{~W}, 15 \mathrm{~s}, 41^{\circ}, \sim 11 \mathrm{~mL} / \mathrm{h}\right)$ short $(12-15 \mathrm{~s})$ ablation (FlexAbility-D-SE) at this area was performed and isolation of the superolateral wall achieved including the left atrial appendage (Fig. 10). High-power 
short duration ablation $\left(70 \mathrm{~W}, 8 \mathrm{~s}, 41^{\circ}, 11 \mathrm{~mL} / \mathrm{h}\right)$ was then extended to the area of the inferoseptal left atrial ganglia, where fractionated electrograms were detected and abolished (Fig. 11). Substrate mapping was also carried on in the right atrium where an area of fractionated electrograms along the septal border of the superior to inferior vena cava line was found and ablated $\left(6 \mathrm{~s}, 60 \mathrm{~W}, 41^{\circ}\right.$, Fig 12). At the end of the procedure, only short runs ( $<3 \mathrm{~s})$ of $A F$ could be induced with aggressive pacing under orciprenaline infusion. Total RF,X-ray and procedural time were $14,4.5$ and $115 \mathrm{~min}$, respectively. The X-Ray dose was $61 \mu \mathrm{G} / \mathrm{m} 2$. So far, the patient has had no further episodes of symptomatic or asymptomatic (according to EGM monitoring from PM) AF (0,1 millisievert) detected.



Figure 11. Sinus rhythm left atrial voltage map and radiofrequency ablation lesions at redo procedure. Low voltage $(0.5-0.05 \mathrm{mV})$ of the pulmonary veins, the posterior wall and the superior septum in the left lateral view is shown. Achievement of isolation (FlexAbility, $60 \mathrm{~W} / 15 \mathrm{~s}$ ) of the superolateral left atrial wall is proved by left atrial appendage isolation (disappearance of electrograms at the 6th beat on the Advisor FL Circular mapping catheter). In the right posterolateral view, radiofrequency ablation lesions ( $70 \mathrm{~W}, 8 \mathrm{~s}$ ) of complex fractionated electrograms delivered in the area of the inferoseptal ganglia are shown. The Advisor FL Circular mapping catheter is placed in the right inferior pulmonary vein and shows electrical isolation. Inquiry catheter in the coronary sinus (green) and at the His (yellow).

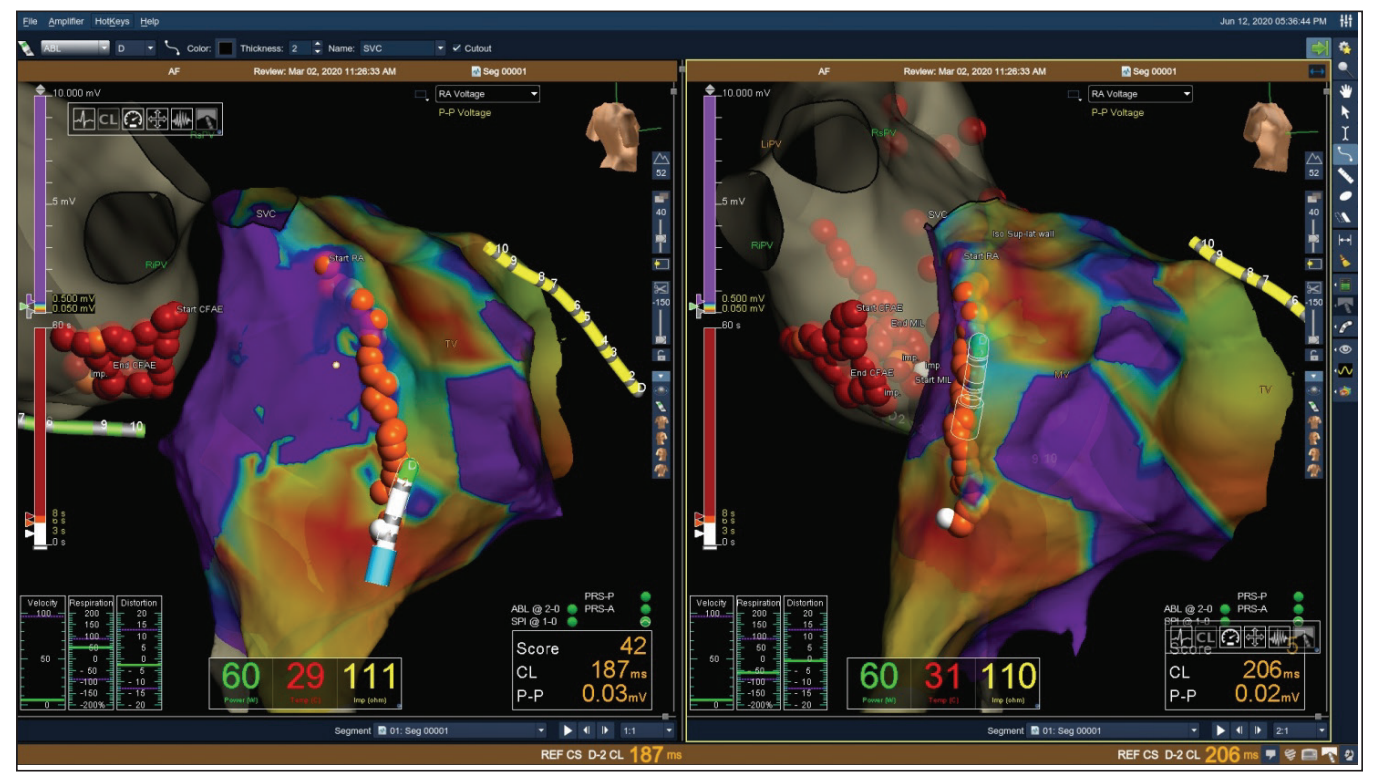

Figure 12. Sinus rhythm right atrial voltage map and radiofrequency ablation lesions at redo procedure. Additional ablation $(60 \mathrm{~W} / 8 \mathrm{~s}$ ) of fractionated electrograms septal of the line (low-voltage area, 0.5-.0.05 mV) on the posterior wall (standard pacing maneuver showed bidirectional block) was performed. Inquiry medium curve (green) in the coronary sinus. Right posterolateral (left) and right anterior oblique view $30^{\circ}$ (right) views are shown. 


\section{DISCUSSION}

The excellent long-term results of the full biatrial Cox-Maze procedure, an approach supported by the randomwavelet theory of $\mathrm{AF}$ sustenance, provide the rationale for extensive left and right linear catheter ablation in addition to pulmonary vein isolation for the treatment of long-standing persistent AF. In order to appropriately reduce the fibrillatory mass, completeness of any line is key to success. This anatomical approach is combined with a more electrophysiological "substrate-based" strategy from mapping, where lines are specifically plotted in areas of low-voltage complex fractionated EGMs, and in other regions where ablation has often been shown to be associated with AF termination4,5.

The pathophysiological reasoning behind this approach is based on the theory of leading circle reentry for AF sustenance. In case of recurrences, where focal sources cannot be elicited by pharmacological testing, further reduction of the potential fibrillatory mass is pursued. This is most often realized by broad isolation of the left atrial appendage with a "double" inferolateral and superoseptal mitral isthmus line, especially if low-voltage fractionated EGMs are detected (in sinus rhythm) on the contralateral mitral isthmus line (in this case the inferolateral isthmus after a previous successful superoseptal mitral line). Furthermore, the potential proarrhythmic nature of complex fractionated low-voltage electrograms in sinus rhythm is often established with sensed atrial pacing (single or doubles at a CL 300/350 ms).

The disappearance of the late components of fractionated EGMs likely provides proof of inability to sustain any (functional) reentry at higher heart rates (i.e. in AF). On the contrary, persistence of these late components of fractionated EGMs prove that reentry can still be sustained at higher heart rates, and therefore these areas are targeted for ablation.

Therefore, an effective treatment of long-standing persistent atrial fibrillation might be based on atrial debulking of lowvoltage areas (detected either in AF or sinus rhythms) in the form of extensive linear ablation, additionally targeting areas of complex fractionated EGMs which have often been shown to be associated with termination (if ablation is performed during AF) or ability to sustain (functional) reentry as proved by pacing maneuvers (if ablation is performed in sinus rhythm).

\section{AUTHOR'S CONTRIBUTION}

Conceptualization, Brunelli M.; Writing - Original Draft, Brunelli M. and Sammut M. A.; Writing - Review and Editing, Brunelli M. and Sammut M.

\section{REFERENCES}

1. Kirchhof P, Benussi S, Kotecha D, AhIsson A, Atar D, Casadei B, et al. 2016 ESC Guidelines for the management of atrial fibrillation developed in collaboration with EACTS. Eur Heart J. 2016;37(38):2893-962. https://doi.org/10.1093/eurheartj/ehw210

2. Marrouche NF, Brachmann J, Andresen D, Siebels J, Boersma L, Jordaens L, et al. Catheter ablation for atrial fibrillation with heart failure. N Engl J Med. 2018;378(5):417-27. https://doi.org/10.1056/NEJMoa1707855

3. Calkins H, Hindricks G, Cappato R, Kim Y-H, Saad EB, Aguinaga L, et al. 2017 HRS/EHRA/ECAS/APHRS/SOLAECE expert consensus statement on catheter and surgical ablation of atrial fibrillation: Executive summary. EP Europace. 2018;20(1):157-208. https://doi. org/10.1093/europace/eux275

4. Schmitt C, Estner H, Hecher B, Luik A, Kolb C, Karch M, et al. Radiofrequency ablation of complex fractionated atrial electrograms (CFAE): Preferential sites of acute termination and regularization in paroxysmal and persistent atrial fibrillation. J Cardiovasc Electrophysiol. 2007;18(10):1039-46. https://doi.org/10.1111/j.1540-8167.2007.00930.x

5. Nademanee K, McKenzie J, Kosar E, Schwab M, Sunsaneewitayakul B, Vasavakul T, et al. A new approach for catheter ablation of atrial fibrillation: mapping of the electrophysiologic substrate. J Am Coll Cardiol. 2004;43(11):2044-53. https://doi.org/10.1016/j. jacc.2003.12.054 